\title{
Single thermal plume in locally heated vertical soap films
}

\author{
N. Adami, ${ }^{*}$ S. Dorbolo, and H. Caps \\ GRASP, Université de Liège, Physics Department B5, B-4000 Liège, Belgium \\ (Received 18 February 2011; revised manuscript received 29 August 2011; published 19 October 2011)
}

\begin{abstract}
A vertical soap film is maintained by injection of a soap solution from the top. The film is then locally heated. Thermal plumes may be observed to rise in the film, depending on the magnitude of the heating and injected flows. The nearly two-dimensional nature of the system allows to visualize the motion of the plumes using an infrared camera. A model is proposed to describe the growth, emergence, and stationarity of the plumes in the film by taking into account both magnitudes of the heating $\Delta T$ and injected flow $Q$.
\end{abstract}

DOI: 10.1103/PhysRevE.84.046316

PACS number(s): 47.15.gm, 47.55.dm, 47.54.-r

\section{INTRODUCTION}

Being the basic components of a foam, thin liquid soap films have been the subject of numerous studies during the last decades [1-3]. Phenomena, such as foam drainage, are explained by considering the fluid flows in the liquid films and Plateau borders network which constitute a foam [4]. A soap film can be seen as an amount of fluid imprisoned between two free interfaces. The main difference between soap film interfaces and rigid walls resides in the fact that the walls are part of the system itself. Under the influence of gravity, the soap film interfaces get closer or further apart, provoking internal fluid flows, which result from a balance between viscous forces, capillarity, and gravity. This is the socalled free drainage phenomenon $[5,6]$. In opposition, forced drainage refers to the constant feeding of the film with the surfactant solution, leading to steady thickness profiles. Forced drainage is used in order to increase soap films lifetimes, and to produce steady nearly two-dimensional (2D) flows. Numerous studies have been intended for such regimes. They mainly focused on thickness dynamics [7], velocity fields [2], specific phenomena, such as marginal regeneration [8], surface rheology [9,10], and 2D turbulence [11-15]. Even if good insights were brought in the last decades, those topics are still under investigation.

The case of silicone oil films has to be mentioned. The films based on this pure fluid exhibit thickness and velocity profiles which are quite different from those observed in soap films $[16,17]$. These films were used to study the development of a local vertical perturbation in a 2D system due to a mechanical local stress [16,17].

Thermal gradients may be also applied to the soap film. Rayleigh-Bénard-like experiments have been carried out in soap films, in order to investigate $2 \mathrm{D}$ thermal-driven turbulence $[15,18,19]$. Those experiments have revealed that the heating of a soap film leads to the apparition of buoyant upward flows, called thermal plumes, similar to those observed in classical three-dimensional (3D) RayleighBénard systems [15,18-20]. Moreover, in spherical configuration, isolated vortices are observed to produce trajectories which are similar to those of cyclones in the Earth's atmosphere [20].

*n.adami@ulg.ac.be
The thermal plumes' dynamics has been widely emphasized in 2D thermally stressed film in Refs. [15,18-20]. However, to our knowledge, single thermal plumes produced in 2D fluid systems have never been studied. The generation of stationary single thermal plumes in a large soap film is the focus of the present work. The paper is organized as follows. The experimental setup is presented in the next section. We then explain the film feeding and heating procedures. The experimental observations first focus on the film thickness when no thermal gradient is applied. The latter is indeed required to characterize the drainage and the marginal regeneration flows in the film. The existence conditions to obtain a plume and the dynamics of the plume generation are described in Sec. IV and compared to previous works. We eventually conclude in Sec. V.

\section{EXPERIMENTAL SETUP}

Figure 1 is a sketch of the experimental setup used to locally heat soap films. Soap films were created from $1 \%$ surfactant solution [21], whose viscosity was tuned by adding a given amount of glycerol, fixed to $10 \%$ for all the experiments described below. This leads to a kinematic viscosity of $1.09 \times 10^{-6} \mathrm{~m}^{2} / \mathrm{s}$ [22].

To create the soap films, the solution was injected in a horizontal pipe by means of a controlled-flow pump Ismatec BVP70. A slot was grooved in the pipe, in order to allow the solution to flow out of it from the top. Films were sustained by a metallic frame, whose size was fixed to $150 \times 150 \mathrm{~mm}^{2}$ for all the experiments presented here. This kind of setup was used in previous experiments to produce silicone oil thin films $[16,17]$. To locally heat the films, a stainless steel tube crossed perpendicularly the vertical film $20 \mathrm{~mm}$ above the bottom of the frame. A constant flow of thermalized water circulated through the pipe warming locally the soap film (see Fig. 1).

The thermal mapping of the soap film was performed using a FLIR ThermoVision 160 infrared camera (see Fig. 1). In order to get relevant data, the origins of infrared intensities detected by the camera have to be well identified; they originate from transmission through the film, emission by the film, or a sum of both. Being aware of that, the camera can be used according to two different purposes: (i) measuring film thickness profiles, and (ii) getting thermal maps of the heated soap film. 


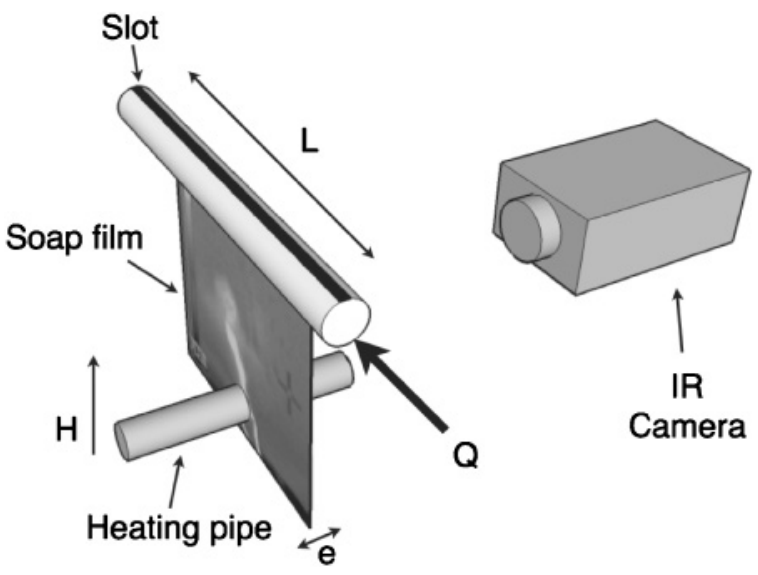

FIG. 1. Experimental setup used to produce thermal plumes in fed soap films. See text for details.

\section{A. Thickness measurement procedure}

Thickness profiles were obtained only when there was no local heating applied to the film. In order to determine the thickness profiles, a thermalized bath was placed behind the films, and the corresponding transmitted infrared intensity was recorded by the camera. The Beer-Lambert law can then be used to get the thickness profile in the whole film:

$$
I(e)=I_{0} e^{-\mu e},
$$

where $I_{0}$ and $I(e)$ are intensities behind and in front of the film, respectively, and $\mu$ is the absorption coefficient linked to the transparent media at a given wavelength. It has been shown $[7,15]$ that infrared reflectance of soap films is negligible in the determination of their transmittance, allowing the film thickness to be determined thanks to Eq. (1). Consequently, the knowledge of $\mu$ allows us to determine the thickness $e$ by inverting Eq. (1). Three conditions are then required to perform this measurement.

The first condition concerns the source of infrared light, i.e., the thermal bath. It should be considered as a black body. In the present experiment, $I_{0}$ corresponds to the infrared intensity emitted by the thermalized bath located behind the film, whose temperature was set to $80^{\circ} \mathrm{C}$. Since water was thermalized in a Pyrex tank, the emissivity linked to the thermal bath is about $97 \%$ [23]. Thus, it can be considered as a perfect blackbody to a first approximation.

The second condition is the determination of the absorption coefficient $\mu$. Since surfactant solutions are $90 \%$ made of water, their absorption coefficients can be approximated by the one of pure water, and determined according to measurements presented in Ref. [24]. However, the $\mu$ coefficient depends on the considered wavelength. A thermal mapping of the thermalized bath has been recorded with the infrared camera allowing us to determine its surface temperature. The corresponding wavelength can be determined using the Wien law, which gives the position of the intensity peak linked to the corresponding blackbody:

$$
\lambda_{\max }=\frac{h c}{4.9651 k_{B} T}=\frac{2.898 \times 10^{-3}}{T},
$$

with $h$ being the Planck constant, $c$ the speed of light, $k_{B}$ the Boltzmann constant, and $T$ the temperature of the blackbody. The typical wavelength linked to the thermal bath and determined by Eq. (2) is close to $8.3 \mu \mathrm{m}$. It is then possible to know the absorption coefficient linked to the intensity peak of the thermalized bath. Even if Eq. (2) gives the wavelength corresponding to the maximal intensity emitted by a blackbody, it can be used to determine $\mu$ from tables giving its dependency on the wavelength [24] due to the fact that $\mu$ remains almost constant in the wavelength range considered in our experiments [24]. This kind of method has already been used with far infrared light, and has proven to be well suited to perform one-point thickness measurements in soap films [7].

The third condition comes from the environment which also emits infrared radiation that can disrupt the measurement. The infrared intensity range displayed by the camera can be fixed by the user. A judicious choice of that thermal range of interest ensures that only the relevant thermal spectra are considered for thickness measurements. Indeed, it is possible to set the intensities emitted by the film due to its natural temperature out of the range of interest. When the selected working range is relevant, any object whose temperature is the same as the temperature of the solution is invisible to the infrared camera.

\section{B. Plume visualization}

The infrared camera was used to visualize thermal maps of the heated films. This is consistent with the thermal emissivity of thin water sheets, which is of the order of $96 \%$ [25]. Contrary to the thickness profile determination, the local fluctuations of the film temperature and/or infrared intensity were emphasized by means of a cold background, which was put behind the heated film. The temperature of the background was fixed at $-10^{\circ} \mathrm{C}$. Using this kind of cold background allows us to enhance the thermal contrast linked to the heated soap films,

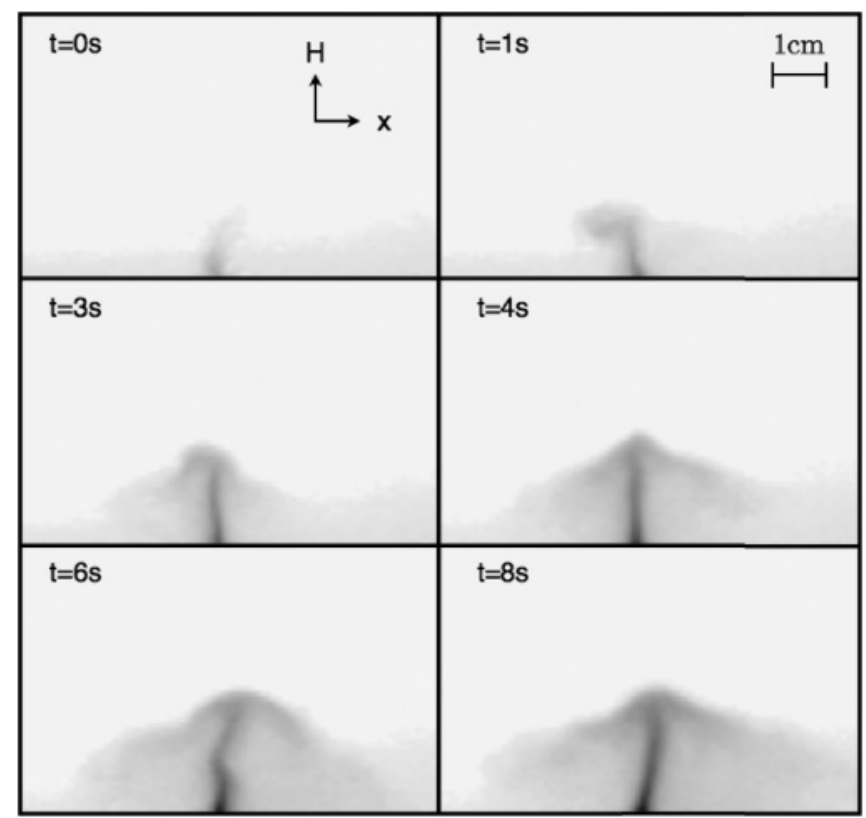

FIG. 2. Infrared images used to investigate thermal plumes' behaviors $\left(Q=0.76 \mathrm{~cm}^{3} / \mathrm{s}, \Delta T=21^{\circ} \mathrm{C}\right)$. The darker the area is, the hotter the temperature is. 
making thermal phenomena appear more clearly on infrared images (see Fig. 2). Since that method only aims in visualizing thermal phenomena conveniently, it was not necessary to know the corresponding wavelength anymore. Again, the Pyrex tank containing the thermal bath has a thermal emissivity of 0.97 [23], which insures the efficiency of the cold background to be optimized.

\section{STUDY OF THE FLOW IN A NONHEATED FILM}

\section{A. Thickness profile}

We first consider soap films without any heating. The purpose is to analyze the thickness profiles of the films without thermal perturbations. As mentioned in the previous section, films are fed thanks to a constant flow pump. Mass conservation implies

$$
Q=e(H) v(H) L,
$$

where $Q$ is the flow delivered by the pump, $H$ is the vertical coordinate taken from the bottom of the sustaining frame [Fig. (1)], $e$ is the film thickness, $v$ is the fluid vertical mean velocity, and $L$ is the frame width. Due to the mass conservation, any change in $Q$ induces a thickness and/or a velocity change.

Numerous experimental and theoretical investigations have been carried out on thickness profiles and dynamics in soap films $[1,2,7]$. They brought out that the thickness profiles strongly depend on the chemistry of the fluid composing the film, as well as on the size of the frame sustaining the film [26]. Figure 3 is a plot of $e(H)$ obtained using Eq. (1) for several values of $Q$. Those curves are averaged over the horizontal coordinate $x$. Each curve is an average over numerous experiments, repeated for each $Q$ value.

Fits to the experimental thickness measurements reveal that a power law is well suited to describe them. Theoretical developments, however, suggest an exponential dependence of the thickness profiles [2], but are discredited by $\chi$-square tests. Thus, $e(H)$ is fitted by

$$
e(H)=a H^{-\beta}+e_{0},
$$

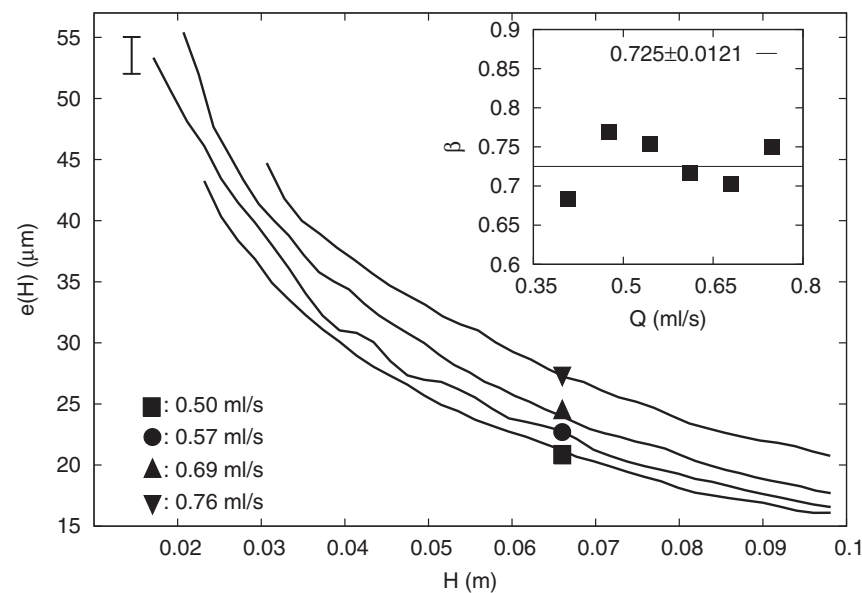

FIG. 3. Film thickness vs vertical coordinate $H$ for several values of $Q$. The inset shows the dependence of the exponent of Eq. (4) on the injected flow. where $a$ is a scaling constant, $\beta$ the scaling exponent describing the decrease of $e$ with $H$, and $e_{0}$ the thickness at the upper limit value considered for $H(0.1 \mathrm{~m}$ in the present case). It should be noted that thickness profiles presented on Fig. 3 only present data a few centimeters away from any edge of the sustaining frame. The thickness in those regions is indeed controlled by the properties of the surfactant, i.e., its capillary length [5]. Equation (4) thus describes the decrease of $e$ with $H$ only in film regions which are not influenced by the menisci linking the film to the sustaining frame.

Thickness profiles displayed in Fig. 3 show that an increase in $Q$ is followed by an increase of $e(H)$, with no crossing between the different curves. The inset of Fig. 3 shows that $\beta$ remains almost constant in the explored $Q$ range. This can be explained by the fact that the injected flows are so small that increasing the $Q$ value means changing the amount of solution arriving in the film per unit time, with no added pressure difference to the hydrostatic pressure. The increase of $e(H)$ with $Q$ simply represents an increase of the amount of fluid imprisoned in the film. The 0.73 mean value for $\beta$ must be regarded as resulting from a balance between hydrostatic pressure in the film and surface tension gradients sustaining the film [6]. According to Eq. (3), this behavior implies that the velocity of the downward flow increases with $H$. It can be underlined that the opposite behavior is observed in silicone oil films [16,17].

\section{B. Marginal regeneration}

In the vicinity of the frame sustaining the film, the so-called marginal regeneration phenomenon $[1,8]$ can be evidenced. Figure 4 presents horizontal thickness profiles close to the sustaining frame. Points close to $x=0 \mathrm{~mm}$ represent the Plateau border. For $x>30 \mathrm{~mm}$, the film is not influenced by the frame anymore. The thickness decrease that occurs between the bulk of the film and the meniscus illustrates the release area of thin film elements from the Plateau border [8]. This production of thinner film elements leads to an upward flow, which rises upward against the drainage flows. Those

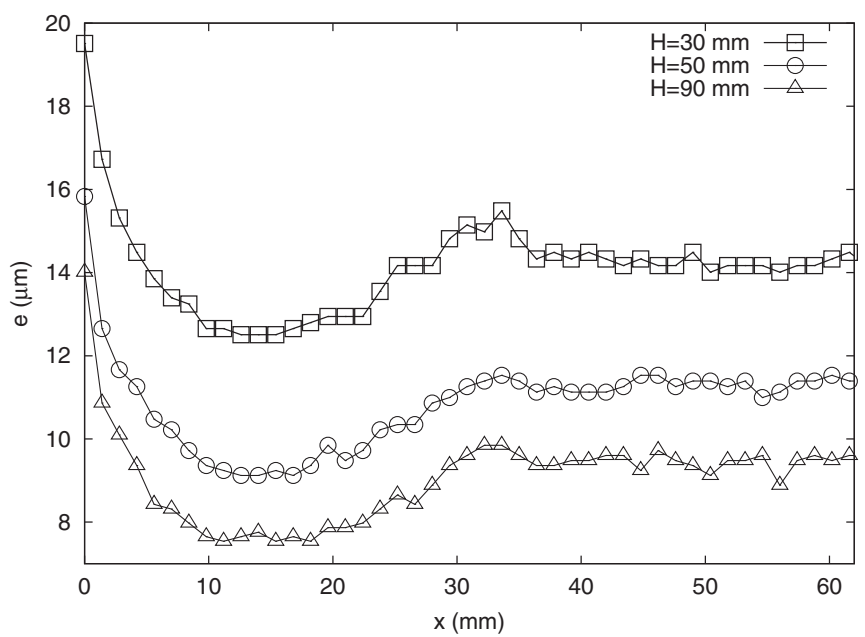

FIG. 4. Thickness profiles as functions of the horizontal coordinate $x$. Those profiles are considered for different values of the vertical coordinate $H$. 
marginal regeneration flows are known to act on the thickness dynamics of soap films [1].

In order to take these marginal regeneration flows into account, mass conservation [Eq. (3)] in the film should be rewritten as

$$
Q=Q_{d}+Q_{r m},
$$

where $Q$ is the net flow injected in the film by the pump, $Q_{d}$ is the effective downward flow in the film, and $Q_{r m}$ is the upward marginal regeneration flow. As described in Ref. [8], this marginal regeneration flow is due to the pressure gradient existing between the Plateau border and the bulk of the film. This gradient is present for every border of the film. However, downward marginal regeneration flows coming from the pipe's vicinity are very small compared to those occurring at the bottom and side edges, due to the fact that thin film elements produced in this area are immediately pushed back up by buoyancy. Infrared visualization of regeneration flows near the frame shows that small thin pieces of soap films are released continuously from the Plateau border. Their typical size and thickness can then be estimated thanks to infrared images (see Fig. 4). If the regenerated particles are assumed to have a cylindrical shape, their typical volume is given by $\pi e^{\prime} l^{\prime 2}$, with $e^{\prime}$ being the height of the cylinder, and $l^{\prime}$ being the radius of its base. The height can be determined from thickness profiles (see Fig. 4) while the radius can be determined by simple infrared or visible light observation of the phenomenon. Moreover, a solid border of size $L$ can release $L / l^{\prime}$ particles during a single marginal regeneration process. $Q_{r m}$ can then be expressed as

$$
Q_{r m}=\frac{3 \pi e^{\prime} l^{\prime 2}}{\tau_{r m}} \frac{L}{l^{\prime}},
$$

where $\tau_{r m}$ is the typical generation time of thin particles. It corresponds to the time which is necessary to bring a film element into the Plateau border and to release a thinner element. A simple dimensional calculation allows us to get an expression for this characteristic time:

$$
\tau_{r m} \sim \frac{\rho v}{\gamma R} l^{2}
$$

where $R$ is the radius of the frame edge, and $l$ the initial distance between the film element and the Plateau border. The proportionality between $\tau_{r m}$ and $v$ seems consistent. The dependence on $R$ is related to the radius of the edge of the frame: the larger the local curvature is locally imposed on the film, the higher the pressure gradient between the Plateau border and the bulk of the film is [8]. The dependency on $\gamma$ can be justified by the thinning of the film as $H$ increases. Mechanical equilibrium considered at a given $H$ means that $\gamma$ must increases with $H$ in order to sustain the weight of the lower parts of the film. Moreover, the thickness gradient between the Plateau border and the bulk of the film increases if the thickness decreases, since they are both fixed by the thickness and the radius of the frame edge. $\tau_{r m}$ is thus expected to decrease with growing $\gamma$.

Considering that the distance between the Plateau border and the bulk of the film is approximately $10^{-2} \mathrm{~m}$ (see Fig. 4) and that the frame edge is $3 \mathrm{~mm}$ in diameter, Eq. (7) brings out that the order of magnitude of $\tau_{r m}$ is $10^{-2} \mathrm{~s}$. Results from Fig. 4 and infrared observations of marginal regeneration show that the typical thickness of an element of the regenerated film is typically $1 \mu \mathrm{m}$ with a lateral extent of about $1 \mathrm{~mm}$. Injecting those values in Eq. (6) gives

$$
Q_{r m} \sim 0.4 \mathrm{~cm}^{3} / \mathrm{s} .
$$

This value is quite close to the magnitude of the injected flow $Q$. This estimation confirms that marginal regeneration flows have to be taken into account.

\section{SINGLE THERMAL PLUME}

\section{A. Thermal gradient criterion}

Figure 2 evidences the rise of thermal plumes in the film as a consequence of the local heating. Their direction opposes the drainage flow. At fixed $Q$, the typical size of plumes increases when the temperature difference $\Delta T$ between the film and the heating source is increased. Moreover, when the temperature difference is fixed, an increase in $Q$ involves a decrease of the plumes' size, as well as an increase of the typical growth time. This suggests that a critical temperature difference $\Delta T_{c}$ has to be applied in order to allow plumes to rise against a given flow. Figure 5 shows the dependence of $\Delta T_{c}$ versus $Q$. Those data are obtained by fixing $Q$ and then varying $\Delta T$ continuously until thermal plumes can be seen in the film. Even for weak values of $Q$, the smallest $\Delta T$ needed is not nought: a temperature threshold exists. Figure 5 shows that $\Delta T_{c}$ increases almost linearly with $Q$ until $0.8 \mathrm{~cm}^{3} / \mathrm{s}$. The same kind of dependency for the critical temperature was previously evidenced both theoretically and experimentally [18]. For higher values, inhomogeneities appear in the downward flow, leading to perturbed velocity fields within the bulk. Those inhomogeneities are due to the appearance of droplets along the border of the pipe, leading to intense mixing in the downward flow. Those perturbations may be responsible for the increase in $\Delta T_{c}$ at high $Q$. In order to avoid those perturbations, the explored range of $Q$ is limited to $0.8 \mathrm{~cm}^{3} / \mathrm{s}$ for all the experiments. The solid line drawn on Fig. 5 is discussed below.

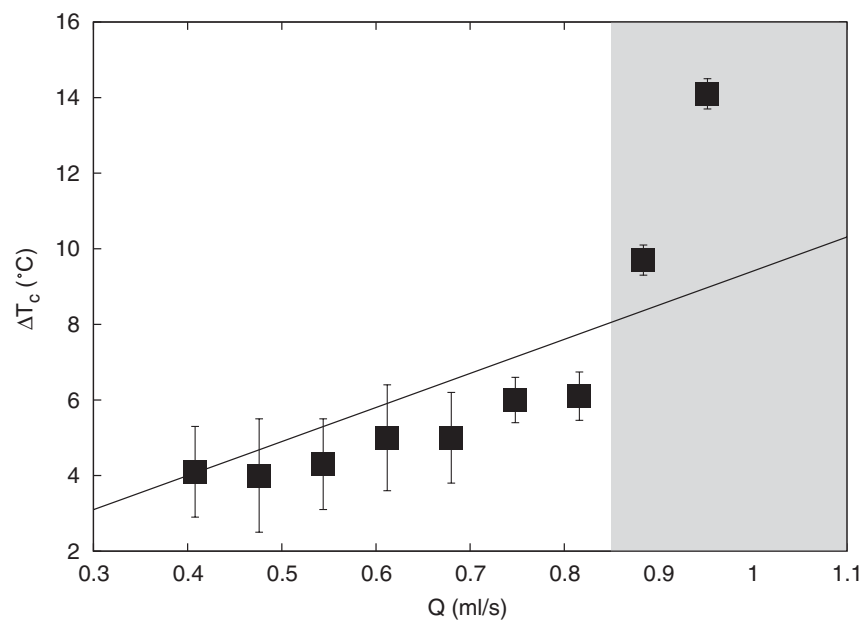

FIG. 5. Evolution of $\Delta T_{c}$ vs $Q$. Points represent experimental values, while the solid line represent a semitheoretical model of $\Delta T_{c}$ [see Eq. (18)]. 


\section{B. Evaluation of the different contributions to the rising flow}

Due to the confined nature of soap films, both thermal buoyancy and Marangoni effects must be taken into account for describing the driving forces responsible for the appearance of thermal plumes. It is observed that once the heating is started, plumes begin to form in the vicinity of the heating point, and then grow until they reach the center of the film. Experimental investigation of 3D cases (Rayleigh-Bénard cells, hot spots in the Earth's mantel, and autocatalytic reactions [27-30]) have identified the thermal buoyancy to be the main effect leading to 3D plumes' growth. In the case of soap films, interfacial thermally driven forces should be considered as an additive effect to thermal buoyancy. However, it can be demonstrated that Marangoni effects are negligible in comparison to thermal buoyancy. Applying a local heating to a horizontal soap film inhibits buoyancy. Infrared observation of such a system shows that no plumes are generated, whatever the $\Delta T$ applied. This experimental proof of the predominancy of thermal buoyancy on the Marangoni effect can be recovered by an order of magnitude calculation. Buoyancy forces magnitude can be estimated by [5]

$$
F_{A} \sim \rho \alpha \Delta T g \Omega,
$$

where $\rho$ is the solution density, $\alpha$ its thermal dilatational coefficient, $\Delta T$ the temperature difference, and $\Omega$ the volume of a typical fluid particle. All those quantities are known or determined by experiments, except $\Omega$. Nevertheless, it can be removed from this expression by expressing Newton's law for a fluid particle of mass $\rho \Omega$. If the particle is assumed to move from the heating point to a height $H$, and that the typical time needed to reach this height due to thermal buoyancy is $\tau_{A}$, the corresponding acceleration can be estimated by $H / \tau_{a}^{2}$, and Newton's law for the particle reads

$$
\rho \alpha \Delta T g \Omega \sim \rho \Omega \frac{H}{\tau_{A}^{2}} \Rightarrow \tau_{A}^{2} \sim \frac{H}{\alpha \Delta T g} .
$$

On the other hand, the capillary time is expressed as

$$
\tau_{\gamma} \sim \sqrt{\frac{\rho H^{4}}{\gamma e}}
$$

where $\gamma$ is the surface tension and $e$ the thickness of the film. This expression can be used to estimate the characteristic time linked to the thermal Marangoni effect. Using typical values of $\gamma\left(10^{-2} \mathrm{~N} / \mathrm{m}\right), \alpha\left(2.6 \times 10^{-4} \mathrm{~K}^{-1}\right), \Delta T\left(1^{\circ} \mathrm{C}\right)$, and $e\left(10^{-5} \mathrm{~m}\right)$, those times take the following values in order to bring a fluid particle to $H \sim 10^{-1} \mathrm{~m}$ :

$$
\tau_{A} \sim 1 \mathrm{~s} \text { and } \tau_{\gamma} \sim 10^{3} \mathrm{~s} .
$$

Those values indicate that thermal buoyancy is more efficient in bringing a heated particle to the top of the film than the Marangoni effect, which confirms experimental observations. It should be noted that previous formal descriptions of heating produced in vertical soap films are in agreement with our results [18].

While the Marangoni effect seems irrelevant in order to describe the large scale motion of the thermal plumes, its local effect on heated particles should be taken into account for describing the thermal plumes' growth. Similarly to the argumentation for the chemical Marangoni effect $[8,26]$, we will consider a 2D density, expressed as $\rho_{2}=\rho e$, with $e$ being the local thickness of the film and $\rho$ the density of the surfactant solution $[15,18,26]$. The idea is that the Marangoni effect due to heating induces a local stretching of film particles, leading to a decrease of the film thickness and then providing buoyancy to the particle through a local diminution of $\rho_{2}$. The slow character of the thickness relaxations compared to velocity relaxations allows those $2 \mathrm{D}$ density fluctuations to be a relevant driving mechanism to describe spontaneous convection in soap films [26]. In fact, it can be easily observed that thin regenerated film elements remain thinner than any other area of the film during their rise, and that thinning lasts until they reach the upper meniscus of the film, or any film area whose thickness is weak enough so that the 2D particle density equals the $2 \mathrm{D}$ density of its surroundings. This phenomenon can be observed along any meniscus induced by the frame or any intrusive object deforming the film, via the marginal regeneration mechanism. As a logical consequence, observation of nonheated films shows that marginal regeneration flows emanate from the heating pipe when no heating is applied.

The variation of $\rho_{2}$ induced by Marangoni stretching can be written as $[15,18,26]$

$$
d \rho_{2}=\rho_{2} \alpha^{\prime} d e,
$$

with $\alpha^{\prime}$ being an effective expansion coefficient expressed as $1 / e(H=0)$ [18], and $d e$ the thickness variation encountered by the ascending particle. In addition to the marginal regeneration-Marangoni component described by Eq. (13), we add a thermal origin to $d \rho_{2}$. In fact, if one considers the capillary time expressed by Eq. (11) for a typical size close to $10^{-4} \mathrm{~m}$, which is the resolution of the camera, and which we assimilate as the typical film particle size, one finds $\tau \sim 10^{-3} \mathrm{~s}$, which implies that the thermal induced Marangoni effect is rather efficient at small scales. We thus decide to express the 2D density fluctuations for the case of our soap films as follows:

$$
d \rho_{2}=\rho_{2} \alpha^{*} d e,
$$

with $\alpha^{*}=\alpha^{\prime} \Delta T / \Delta T_{c}$. When the injected thermal energy increases, the thickness decreases more rapidly. The Eq. (14) is linear regarding to $\Delta T$ and consequently consists in a local linear approximation of a more general expression.

\section{Growth mechanism: criterium}

Figure 6 shows the measured height $H(t)$ reached by the thermal plume as a function of time. After a rapid growth, $H(t)$ fluctuates around an equilibrium value $H^{*}$, which increases with $\Delta T$. The inset of Figure 6 presents the evolution of the aspect ratio of the plumes and the film obtained by dividing $H^{*}$ by the width $L$ of the cell. As shown on this curve, this ratio is found in the range $0.1-0.25$, but never reaches unity. This means that plumes are always smaller than the frame sustaining the film. This is not the case for 3D plumes, whose size is fixed by the dimension of the tank [27,30]. This experimental fact is observed whatever the temperature difference applied. The mass conservation for the entire film [see Eq. (3)] is responsible for this. As explained before, the film thickness $e$ decreases with the height $H$. Thermal plumes' ascension is observed if the driving forces counterbalance the friction 


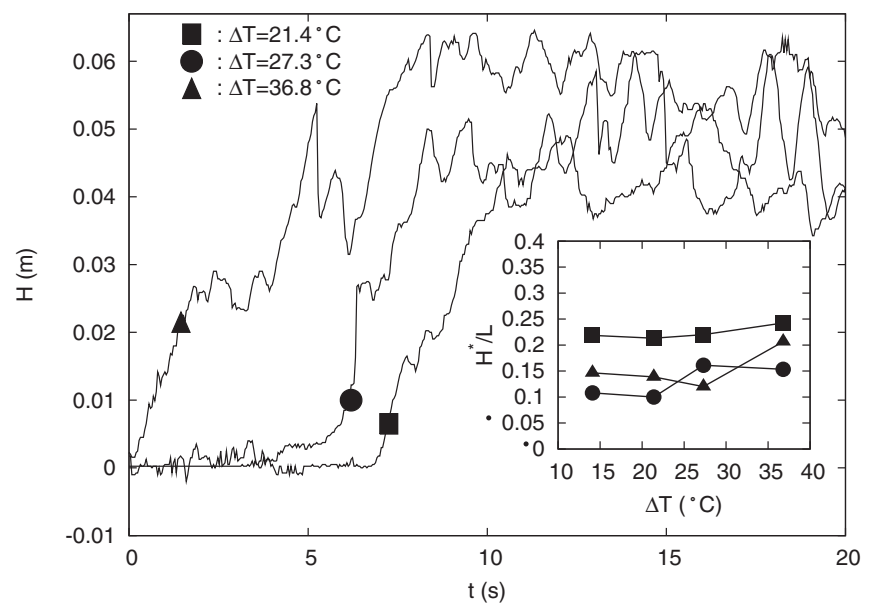

FIG. 6. Height reached by the plume in the film bulk vs time. The injected flow value is fixed at $0.5 \mathrm{~cm}^{3} / \mathrm{s}$. The inset represents aspect ratios linked to different experimental parameters. Squares, circles, and triangles represent flows values of $0.44,0.5$, and $0.57 \mathrm{~cm}^{3} / \mathrm{s}$, respectively.

forces linked to the downward flow. The maximum height $H^{*}$ then indicates the height for which equilibrium is reached. As a consequence of this saturation, the rising velocity is not constant. 3D thermal plumes like those in the Earth's mantel are known to grow with constant speed. Rogers et al. [30] have created autocatalytic plumes which accelerate during their growth, but no decelerating plumes have been observed so far, nor plumes having an equilibrium height smaller than the size of the experimental cell.

\section{Growth mechanism: model}

A hot fluid particle is considered in the vicinity of the heating point in the bottom of the film. Due to thermal buoyancy and local Marangoni stretchings, the particle rises up toward the top of the film. Without downward drainage, the viscous force acting on the particle is proportional to its velocity. However, the particle also experiences the feeding flux $Q$ in the downward direction. Newton's law for the particle motion in the film then reads

$\frac{d^{2} H(t)}{d t^{2}}=g \Delta T\left(\alpha+\frac{d e(H)}{e_{0} \Delta T_{c}}\right)-\frac{v}{\pi l e(H)}\left(\frac{Q_{d}}{e(H) L}+\frac{d H}{d t}\right)$,

where $d e(H)$ is the thickness increment calculated from Eq. (4), $g$ the gravity constant, and $l$ the typical size of a film particle. The first term of the right hand side represents the total buoyancy, while $Q_{d} / e(H) L+d H / d t$ is the relative speed of hot fluid particles with respect to the soap film. Logically, $e_{0}$ is taken as being the capillary length, since there is a meniscus induced by the heating pipe. This model assumes that the temperature of the particle is constant during all its ascending motion. This is consistent with experimental observations, since (i) infrared intensity of plumes remains almost constant during their rising and (ii) thermal quantities like thermal diffusivity can be considered as equal to that of water, since solutions are made of $90 \%$ water. The thermal diffusivities of water and air are $1.4 \times 10^{-7} \mathrm{~m}^{2} / \mathrm{s}$ and $2 \times 10^{-5} \mathrm{~m}^{2} / \mathrm{s}$, respectively. Characteristic times for heat diffusivity in the solution and ambient air can be determined as

$$
\tau_{\kappa_{\mathrm{film}}} \sim \frac{H^{2}}{\kappa_{\mathrm{film}}} \sim 10^{5} \mathrm{~s} \text { and } \tau_{\kappa_{\mathrm{air}}} \sim \frac{H^{2}}{\kappa_{\mathrm{air}}} \sim 10^{3} \mathrm{~s} .
$$

Those times are much more important than the time linked to the thermal plumes' growth (see Fig. 6), which confirms that thermal losses by diffusion are negligible during the particle ascension.

Dimensional calculation can be performed with Eq. (15) in order to check its relevancy. Typical injected flows are of the order of $Q \sim 10^{-7} \mathrm{~m}^{3} / \mathrm{s}$. The viscosity is close to $v \sim 10^{-6} \mathrm{~m}^{2} / \mathrm{s}$, and the width of the frame is $L \sim 10^{-1} \mathrm{~m}$. The typical size of the fluid particle $l$ is the most difficult quantity to evaluate. Since the spatial resolution of measurements is defined by the size of the sensor, the maximal spatial resolution is $10^{-4} \mathrm{~m}$, and corresponds to the smallest soap film amount which can be analyzed by the camera. Considering that the growth time is of the order of $1 \mathrm{~s}$ (see Fig. 6), and that the thickness of the film is of the order of $e \sim 10^{-5} \mathrm{~m}$ at the maximal plumes' height $H^{*}$, we can do an order of magnitude calculation by expressing the first and second order derivatives of $H$ as

$$
\frac{d^{2} H}{d t^{2}} \sim \frac{H}{\tau^{2}} \text { and } \quad \frac{d H}{d t} \sim \frac{H}{\tau},
$$

where $\tau$ is the characteristic growth time. Introducing these estimates in Eq. (15), we obtain $H^{*} \sim 10^{-2} \mathrm{~m}$. This calculation is in good agreement with curves displayed on Fig. 6. The critical temperature difference $\Delta T_{c}$ needed to generate a plume in a film can also be estimated. This can be achieved by considering that when the heating is turned on, the speed of the fluid particle must be zero. Since no rise has occurred yet, no thickness variation has been encountered, and $d e(H)=0$. This leads to the following expression for the critical temperature difference

$$
\Delta T_{c}=\left(\frac{v Q_{d}}{\pi l L e^{2}}+\frac{H}{\tau^{2}}\right) \frac{1}{g \alpha},
$$

where $Q_{d}$ is the effective downward flow, $e$ the thickness value in the vicinity of the heating pipe, and $H / \tau^{2}$ the acceleration of the particle. This latter can be estimated from infrared images: hot particles take $1 \mathrm{~s}$ to get $10^{-3} \mathrm{~m}$ away from the pipe. Including those values in Eq. (18) brings $\Delta T_{c} \sim 1^{\circ} \mathrm{C}$, which is in good agreement with results presented in Fig. 5. The linear dependency of $\Delta T_{c}$ is represented by the solid line of Fig. 5, showing a rather good agreement with experimental data until $0.8 \mathrm{~cm}^{3} / \mathrm{s}$. As emphasized previously, values of $Q>$ $0.8 \mathrm{~cm}^{3} / \mathrm{s}$ should be regarded with special care. As already mentioned in the previous section, experiments involving global heating of soap films exhibit similar dependencies for the critical temperature difference linked to thermal flows in soap films [18].

Since all the relevant quantities of Eq. (15) are known, it is possible to numerically solve this modeling, in order to compare numerical and experimental data. A fourth order Runge-Kutta method is applied to Eq. (15). Figure 7 compares numerical and experimental $H(t)$ for three different values of $\Delta T, Q$ being fixed to $0.5 \mathrm{~cm}^{3} / \mathrm{s}$. Those curves exhibit good 

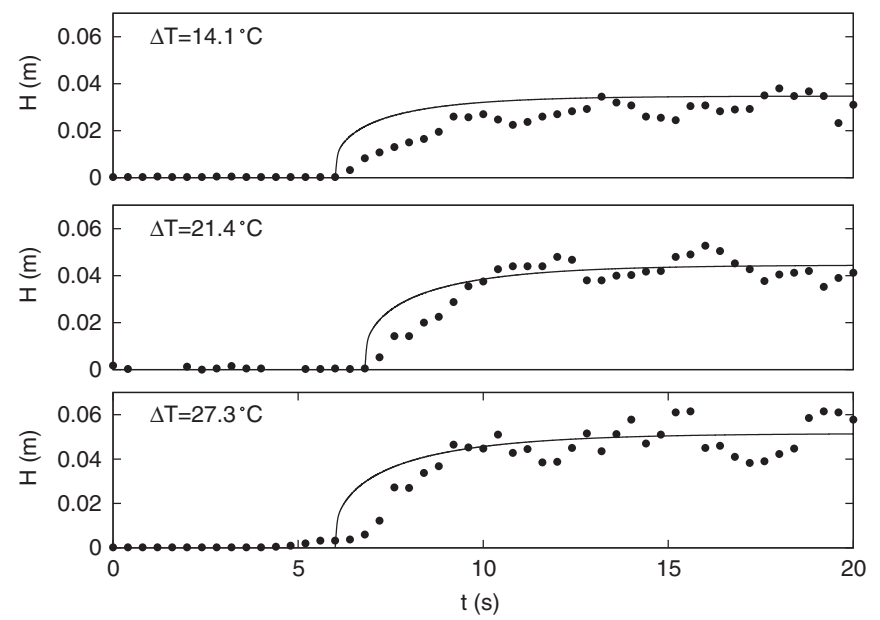

FIG. 7. Superposition of experimental $H(t)$ (points) and numerical results obtained by solving Eq. (15) (solid line). Numerical equilibrium heights and growth times are in quantitative agreement with experimental data.

agreement between numerical and experimental data. Both $H^{*}$ and growth times are rather well described by the model, as well as the critical temperature difference $\Delta T_{c}$. These results justify the assumption made in Eq. (14) regarding the thermal dependence of the interfacial, namely 2D, buoyancy implied by local Marangoni stretching.

\section{E. Comparison with previous models}

The present study aimed to describe the growth and emergence of single thermal plumes in locally heated liquid soap films. Our goal was to predict the characteristics of those plumes (equilibrium height, growth time, etc.) as functions of the experimental parameters, what is well described by Eq. (15). More formal descriptions of similar experiments have been made by Martin et al. [18]. Their experiment aimed to perform a global heating of the soap film, i.e., applied all along its width. Those kinds of Rayleigh-Bénard-like experiments showed that when the temperature difference $\Delta T$ is increased, an oscillatory instability started to develop, leading to wave patterns with a well-defined wavelength and period. When $\Delta T$ is increased again (until $45^{\circ} \mathrm{C}$ ), then a stationary instability develops in the whole film, leading to strongly turbulent behaviors [15]. A formal treatment of those experiments, including a linear stability analysis procedure of adapted fluid dynamics equations (see [18]), brought out that the oscillatory and stationary instabilities were expected to occur for Rayleigh number values of $1.8 \times 10^{4}$ and $5 \times 10^{6}$, respectively. The critical Rayleigh number $(\mathrm{Ra})$ to reach in order to produce thermal plumes in our soap films can be evaluated as:

$$
\mathrm{Ra}=\frac{g \alpha \Delta T_{c} H^{3}}{\kappa \nu} \sim 4.210^{4},
$$

which is quite close to the value which corresponds to the onset of the oscillatory instability described in Ref. [18]. This is not that surprising, since driving phenomena are the same for both experiments. The ratio between values linked to both experiments can be understood by recalling that our films are continuously fed, which tends to enhance the frictions in comparison with a free drainage state. The Rayleigh number associated with fully developed plumes can be evaluated the same way; one finds $\mathrm{Ra} \sim 8.4 \times 10^{6}$ for the smallest temperature difference considered herein. Again, this value is in agreement with the onset of the plume-made stationary instability described in Refs. [15,18].

\section{CONCLUSIONS}

Single thermal plumes were produced in a soap film. A designed setup was used to maintain and drive their inner drainage flows, and to apply controlled local temperature constraints. Infrared imaging of such experiments has shown that thermal plumes may rise in the film due to the heating. Thermal buoyancy, as well as local Marangoni stretching, have been identified to be the driving phenomena leading to the thermal plumes' rise. Despite its local influence, the Marangoni effect has proven to be inefficient to bring heated particles to move on scales larger than the typical size of film particles. For a given magnitude of the drainage flow, a critical temperature difference has to be applied to permit heated film particles to counterbalance viscous friction forces. A model has been built to describe the growth of thermal plumes, taking into account a balance between global buoyancy and viscous friction into the film. Its numerical resolution has proven that this model is well suited to reproduce equilibrium height and growth time which are observed experimentally. Finally, our results are in good agreement with previous formal investigations of similar earlier experiments.

\section{ACKNOWLEDGMENTS}

N.A. and S.D. thank FRS-FNRS for financial support. Part of this work is also supported by ESA MAP Contract No. AO-99-108, ULg fonds spéciaux grant, and FNRS-FRFC No. 2.4558.10.
[1] K. J. Mysels, K. Shinoda, and S. Frankel, Soap Films, Studies of Their Thinning (Pergamon, New York, 1959).

[2] Y. Couder, J. M. Chomaz, and M. Rabaud, Physica D 37, 384 (1989).

[3] H. Kellay and W. I. Goldburg, Rep. Prog. Phys. 65, 845 (2002).

[4] D. Weaire and S. Hutzler, The Physics of Foams (Oxford University Press, Oxford, 1999).
[5] E. Guyon, J. P. Hulin, and L. Petit, Hydrodynamique physique (EDP Sciences, Paris, 2001).

[6] P.-G. de Gennes, F. Brochard-Wyart, and D. Queré, Gouttes, bulles, perles et ondes (Belin, Paris, 2002).

[7] X. L. Wu, R. Levine, M. Rutgers, H. Kellay, and W. I. Goldburg, Rev. Sci. Instrum. 72, 5 (2001). 
[8] V. A. Nierstrasz and G. Frenz, J. Colloid Interface Sci. 215, 6226 (1999).

[9] B. Scheid, J. Delacotte, B. Dollet, E. Rio, F. Restagno, E. A. Van Nierop, I. Cantat, D. Langevin, and H. A. Stone, Europhys. Lett. 90, 24002 (2010).

[10] H. A. Stone, S. A. Koehler, S. Hilgenfeldt, and M. Durand, J. Phys. Condens. Matter 15, 283 (2003).

[11] X. L. Wu, W. I. Goldburg, and M. A. Rutgers, Physica A 239 , 340 (1997).

[12] W. I. Goldburg, A. Belmonte, X. L. Wu, and I. Zusman, Physica A 254, 231 (1998).

[13] M. Rivera, P. Vorobieff, and R. E. Ecke, Phys. Rev. Lett. 81, 1417 (1998).

[14] M. A. Rutgers, Phys. Rev. Lett. 81, 2244 (1998).

[15] J. Zhang, X. L. Wu, and N. Rashidnia, Phys. Fluids 18, 085110 (2006).

[16] J. S. Roche, N. Le Grand, P. Brunet, L. Lebon, and L. Limat, Phys. Fluids 18, 082101 (2006).

[17] N. Le Grand-Piteira, P. Brunet, L. Lebon, and L. Limat, Phys. Rev. E 74, 026305 (2006).

[18] B. Martin and X. L. Wu, Phys. Rev. Lett. 80, 1892 (1998).
[19] J. Zhang, X. L. Wu, and K-Q. Xia, Phys. Rev. Lett. 94, 174503 (2005)

[20] F. Seychelles, Y. Amarouchene, M. Bessafi, and H. Kellay, Phys. Rev. Lett. 100, 144501 (2008).

[21] K. Golemanov, N. D. Denkov, S. Tcholakova, M. Vethamuthu, and A. Lips, Langmuir 24, 9956 (2008).

[22] R. C. Ernst, C. H. Watkins, and H. H. Ruwe, J. Phys. Chem. 40, 5 (1936).

[23] FLIR Thermovision ${ }^{\mathrm{TM}} 160$ user's guide.

[24] G. M. Hale and M. R. Querry, Appl. Opt. 12, 555 (1973).

[25] W. L. Wolfe and G. J. Zissis, The Infrared Handbook (Office of Naval Research, Department of the Navy, Washington, DC, 1985).

[26] R. Bruinsma, Physica A 216, 59 (1995).

[27] E. Moses, G. Zocchi, and A. Libchaber, J. Fluid. Mech. 251, 581 (1993).

[28] B. R. Morton, G. Taylor, and J. S. Turner, Proc. R. Soc. A 234, 1 (1956).

[29] J. S. Turner, Buoyancy Effects in Fluids (Cambridge University Press, Cambridge, 1973).

[30] M. C. Rogers and S. W. Morris, Phys. Rev. Lett. 95, 24505 (2005). 